\title{
AUTOCONCEPTO Y FACTORES DE PROTECCIÓN ASOCIADOS AL CONSUMO DE ALCOHOL Y TABACO EN UNIVERSITARIOS
}

\author{
Self -concept and protection factors associated with alcohol and tobacco \\ consumption among university students
}

\author{
Yanina Sandoval Guerrero*
}

\begin{abstract}
Resumen
Se analiza el autoconcepto y los factores de protección asociados al consumo de alcohol y tabaco en estudiantes de diferentes universidades de Lima. Se empleó un diseño no experimental, de tipo correlacional multivariante. La muestra comprendió a 730 estudiantes de pregrado, del primero al quinto año de estudios, de ambos sexos, con edades entre los 16 y 35 años. Los instrumentos empleados fueron, la escala de autoconcepto de García y Musitu (AF5), Cuestionario de Factores de Protección y una Encuesta de datos sociodemográficos, que además integra preguntas acerca del consumo de alcohol - tabaco. Los resultados indican que los universitarios se iniciaron en el consumo de las drogas mencionadas, entre los 8 y 9 años, presentando la tasa más alta de prevalencia entre los 13 y 17 años (71.2\%). Para quienes consumieron alguna vez en su vida, se encontró que $46.2 \%$ consumían alcohol en el último mes de aplicada la encuesta y 30.8\%, consumían tabaco. Si bien se encontró que el $53.8 \%$ de los estudiantes presentaban un autoconcepto moderado, la cuarta parte de la muestra presentaba un nivel bajo.

Finalmente, el análisis de regresión múltiple permite sostener que el autoconcepto es el factor de mayor capacidad explicativa en el consumo de alcohol $(F=14.438, p<.013)$ y la valoración del cuerpo y de la vida en el consumo de tabaco $(F=8.494, p<.013)$; lo cual quiere decir que a mayor autoconcepto positivo, menor es el riesgo del consumo de alcohol, y que a mayor valoración del cuerpo y de la vida es menor la posibilidad de consumo de tabaco.
\end{abstract}

Palabras clave: Autoconcepto, factor de protección, prevalencia, consumo de alcohol y tabaco.

\begin{abstract}
This article analyzes the self-concept and the protection factors associated to the alcohol and tobacco use in students of different universities of Lima. A not experimental design, of multivariante correlation type was used. The sample composed by 730 undergraduate female and male students, within the first and fifth year of studies, aged between 16 and 35 years. The instruments were the Self-Concept Scale of Garcia \& Musitu (AF5), the Questionnaire of Protection factors and a Survey of information socio demographics data, which also includes questions about alcohol and tobacco use. The results indicate that the university students consumed tobacco and alcohol when they were 8 and 9 years old, presenting the highest prevalence rate between the age of 13 and $17(71.2 \%)$. The people who consumed it sometimes in their life, 46.2 consumed alcohol in the last month that the survey was applied and 30.8\% consumes tobacco. Although it was found that $53.8 \%$ of the students presented a moderate self-concept, the fourth part of the sample showed a low level. Finally, the multiple regressions analysis allows to support that the self-concept is the factor of highest explanatory power for alcohol use $(F=14.438, p<.013)$ and importance of the body and life in the tobacco use $(F=8.494, p<.013)$, which means that if the positive self-concept goes up, the risk of consumption of alcohol goes down, and that if the importance of the body and life is higher, the possibility of consumption of tobacco is lower.
\end{abstract}

Key words: self-concept, protection factor, prevalence, consumption of alcohol and tobacco.

* Magíster en Psicología con mención en Psicología de la Salud. Candidata al Doctorado en Psicología por la Universidad Femenina del Sagrado Corazón. Lima - Perú. yavisan@hotmail.com 


\section{INTRODUCCIÓN}

Uno de los problemas más relevantes en la actualidad, es el consumo de drogas en general, y, de manera específica, de las llamadas drogas sociales como son el alcohol y el tabaco (cigarrillos). En nuestro país los datos que se reportan sobre el abuso y la dependencia a estas sustancias resultan alarmantes, pues los indicadores evidencian que los programas preventivos son escasos, o de existir, son inefectivos. Los jóvenes que refieren consumo de las drogas legales además de ser muy vulnerables al consumo de drogas ilegales como marihuana o derivados de la cocaína, son más propensos al deterioro en su bienestar psicológico, conflictos familiares, bajo rendimiento académico, entre otros.

La universidad como contexto físico y social es un espacio donde se tiene que generar conciencia respecto a las consecuencias de las drogas, y que las llamadas drogas lícitas o sociales están causando consecuencias negativas en la población. Una de las maneras de lograr estos objetivos es hacer una lectura específica sobre la realidad universitaria con respecto a los factores que condicionan o posibilitan el uso del alcohol y el tabaco, así como la de proveer modelos explicativos de los factores psicológicos y contex tuales que cumplen función reguladora o moduladora de los estados emocionales y disposicionales de protección o riesgo frente al consumo y abuso de dichas sustancias y de ese modo, finalmente, poder diseñar y establecer programas de promoción de estilos de vida saludable y bienestar general para los estudiantes universitarios.

\section{ANTECEDENTES DE LA INVESTIGACIÓN}

El Centro de Información y Educación para la Prevención del Abuso de Drogas (CEDRO, 1993) señala en sus resultados la presencia de mayor prevalencia de consumo de tabaco en la población universitaria, pareciendo incluso tener una relación positiva con el nivel de instrucción, presentándose en quien tiene instrucción superior un $83 \%$; secundaria un $67 \%$; primaria $42,5 \%$ y sin instrucción en un $28,1 \%$ respectivamente.

En cuanto al alcohol se ha señalado que es la droga psicoactiva de mayor consumo, y la causante de trastornos biológicos, sociales, familiares, económicos y psicológicos; consumo que se ha incrementado como lo reporta el estudio epidemiológico de drogas en la población peruana (CEDRO, 1997).

Según el estudio epidemiológico metropolitano en salud mental del año 2002, reportado por el Instituto especializado de salud mental "Honorio Delgado - Hideyo Noguchi", las sustancias más consumidas son el alcohol y el tabaco, habiéndose iniciado dicho consumo entre los 12 y 13 años (Saavedra, Nizama, y Kendall, 2006).

El autoconcepto, al igual que las variables de protección, se construye y define por la influencia de las personas significativas del medio familiar, educativo y social, y como consecuencia de las propias experiencias de éxito y de fracaso. El concepto de uno mismo cumple varias funciones en el comportamiento humano, según Burns (1979 y 1982, citado por Machargo, 1991).

Existen estudios realizados sobre la presencia de variables de protección en el campo de la prevención de la drogodependencia. Martínez y Robles (2001) concluyeron en una investigación que las variables "apego familiar, el hecho de valorar la salud", y "el cumplimiento de leyes-normas de la comunidad" parecen proteger a los sujetos de determinadas edades en el consumo de drogas como el alcohol y el tabaco. En otro estudio Muñoz-Rivas y Graña (2001) confirmaron que los principales factores de riesgo familiares para explicar el consumo de drogas legales eran la ausencia de normas familiares sobre el uso de drogas, los conflictos entre los padres y los adolescentes y el consumo de alcohol por parte del padre. En sentido opuesto los factores de protección más importantes eran, acostarse a una hora fija por la noche y tener una buena relación de vínculo con los padres.

La definición de autoconcepto se define como las percepciones que el individuo tiene de sí mismo o bien como la totalidad de pensamientos y sentimientos del individuo con referencia a sí mismo (Tomás y Oliver, 2004).

\section{EL AUTOCONCEPTO COMO FACTOR PROTECTOR EN EL CONSUMO DE SUSTANCIAS PSICOACTIVAS}

De acuerdo a Machargo (1991) el autoconcepto cumple 5 funciones en términos de factor protector: 
Mantiene la consistencia interna; actúa como marco de referencia; regula la conducta; actúa como mediatizador ante el feedback recibido de otra persona; y satisface algunas necesidades básicas como el mantenimiento de la autoestima.

\section{FACTORES PSICOSOCIALES}

Los factores psicosociales están referidos a un conjunto de características o atributos psicológicos y condiciones de tipo social que permiten explicar o predecir el comportamiento de los sujetos en su interacción social.

Dentro de las variables psicosociales a evaluar en la presente investigación, que podrían cumplir función de factores protectores se consideran:

1. El apego familiar, este es considerado como el vínculo afectivo entre padres e hijo.

2. La existencia de normas de conducta claras tanto en el entorno familiar como en la escuela, la disciplina en la familia como en la escuela son variables asociadas a la etiología del abuso de drogas en la adolescencia.

3. Relaciones familiares satisfactorias a favor de la estabilidad y la cohesión familiar, el problema de la conducta adictiva se suele relacionar con la desintegración de la estructura familiar. Muy a menudo tanto el padre como la madre solo se interesan en lo que ellos hacen, sin importarles mucho las actividades del resto de la familia, según Bragado, Bersabe y Carrasco (1999, citado por Muñoz-Rivas y Graña, 2001).

4. Alto apoyo social, el grupo social en el que se desarrolla un individuo es significativo, este puede ser una variable de riesgo como una variable de protección si existen condiciones que permitan al sujeto sentirse parte de un grupo, sentirse aceptado y apreciado por dicho grupo. Además, como refieren muchos autores (Muñoz-Rivas y Graña, 2001) la práctica y asistencia a las actividades religiosas constituyen un factor protector.

\section{Los factores protectores}

Un factor de protección es un atributo o característica individual, condición situacional que inhibe, reduce y atenúa la posibilidad o probabilidad del uso y abuso de drogas (Vallejos, 2004).

\section{Alcohol y Alcoholismo}

El alcohol es la droga psicoactiva legal y social más antigua. Su nombre científico-químico es Etanol y su composición es C4H6O2 (Tapia, 2001). El alcoholismo es también conocido como el Síndrome de Dependencia al Alcohol. La Organización Mundial de la Salud en 1952 (véase Bringas, 2002) definía de la siguiente manera a las personas que consumen alcohol en exceso:

Alcohólicos son todos aquellos bebedores excesivos cuya dependencia al alcohol ha alcanzado un alto grado y que por tanto presentan notables trastornos mentales o interferencias con su salud mental, sus relaciones interpersonales y su funcionamiento social económico. Es por esto que estas personas necesitan tratamiento... (p. 53)

\section{Tabaco y Tabaquismo}

El uso del tabaco se remonta a varios siglos antes del descubrimiento de América. Primero lo utilizaron nuestros antepasados con fines religiosos, y ahora es consumido por millones de seres humanos a pesar de que se sabe que su consumo es perjudicial para la salud y economía familiar (Kuri, 1994).

\section{MÉTODO}

La investigación es de naturaleza correlacional multivariante (Hernández, Fernández y Baptista, 1998). El diseño correspondiente de acuerdo con Kerlinger y Lee (2002) es naturalista o ex-post-facto.

\section{Participantes}

La población la constituye los alumnos del pregrado matriculados en el calendario académico 2009 de las universidades nacionales y particulares de Lima Metropolitana, con una muestra no probabilística de 739 participantes.

\section{Instrumentos}

- Escala de Autoconcepto Forma 5 de García y Musitu (AF5).

Este autoreporte fue construido por García y Musitu (1999) para evaluar el autoconcepto de manera global y de manera específica en cinco dimensiones: Académico-laboral, Social, Emocional, Familiar y Físico. 
- Cuestionario de Factores de Protección:

Este instrumento diseñado por Martínez \& Robles (2001) está constituido por 21 reactivos. Evalúa cinco grandes factores: 1) el apego (apoyo) familiar, 2) la existencia de normas de conducta en el entorno familiar y en la escuela (conocimiento y cumplimiento de normas familiares y escolares), 3) relaciones familiares satisfactorias a favor de la estabilidad y la cohesión familiar (satisfacción familiar), 4) apoyo social, y 5) valores positivos con respecto al cuerpo y a la vida (valoración del cuerpo y la vida).

- Encuesta de datos Sociodemográficos y Consumo de Alcohol - Tabaco:

Es un autoreporte diseñado ex profeso para propósitos de la presente investigación. Contiene 13 reactivos, que indagan acerca de datos personales, consumo de alcohol y tabaco del encuestado.

\section{Procedimiento}

El proceso de la investigación consistió en 2 etapas:

Primera etapa: Se procedió a la adaptación de la escala de autoconcepto de García y Musitu (AF5) como para la población de estudio. Luego, se procedió a la estimación de las propiedades psicométricas (confiabilidad, validez y baremos) para lo cual se empleó una muestra de 350 sujetos con características similares a la población de estudio, de la muestra mencionada.

Segunda etapa: Consistió en la aplicación de los instrumentos a una muestra de 730 sujetos, el análisis preliminar de los datos ausentes y atípicos existentes en los instrumentos llevó a la supresión de 25 cuestionarios, lo que determinó una muestra definitiva de 705 casos para el análisis de los objetivos propuestos en la investigación.

Una vez revisado y depurado los instrumentos se diseñó una base de datos para realizar todos los análisis estadísticos pertinentes con el paquete SPSS 15.0 para Windows. Los análisis descriptivos y de prevalencia fueron establecidos por estadísticos de posición central y análisis de frecuencia; para estimar las relaciones lineales simples se utilizó el estadístico "r" de Pearson; para determinar el factor de mayor peso explicativo tanto para consumo de alcohol como de tabaco se empleó el Análisis de Regresión Múltiple; y para identificar los Odds Ratio (OR) que establecen los factores de protección asociados al consumo de alcohol y tabaco se utilizó el Análisis de Regresión Logística.

\section{Variables de relación}

Autoconcepto: Puntuaciones obtenidas en la Escala de Autoconcepto Forma 5 de García y Musitu (1999).

- Factores protectores: Puntuaciones obtenidas en el Cuestionario de Factores de Protección de Martínez y Robles (2001).

Comprendelos siguientesfactorespsicosociales: apoyo familiar, conocimiento y cumplimiento de normas familiares y escolares, satisfacción familiar, apoyo social, valoración del cuerpo y la vida.

- Consumo de Alcohol - Tabaco: Encuesta de datos sociodemográficos.

\section{RESULTADOS}

La edad de inicio en el consumo de alcohol y tabaco se presenta a partir de los 8 y 9 años respectivamente. Entre los 13 y 17 años es la edad de mayor iniciación en el consumo, tanto de cigarrillos como de bebidas alcohólicas, en la misma proporción. Entre los 18 y 25 años, etapa que comprende a la población universitaria en gran porcentaje, se observa un ligero incremento en el inicio de consumo de bebidas alcohólicas.

Sobre la base de una muestra total (705 universitarios), la sustancia más consumida en alguna etapa de su vida por los universitarios es el alcohol $(85.1 \%)$, y en segundo lugar el tabaco (69.1\%). Esta tendencia se mantiene en cuanto a la prevalencia del último mes, pues los estudiantes admiten que durante el último mes la sustancia que más consumieron fue alcohol (46.2\%).

La gran mayoría de hombres admiten haber probado tabaco $(78 \%)$ y alcohol $(88 \%)$ alguna vez en su vida, igualmente, los estudiantes que provienen de padres separados indican un alto índice de consumo (87\% y $92.5 \%$ respectivamente).

En los grupos etarios de la población universitaria existe una variación con respecto a los que consumen alcohol y tabaco, el grupo de 21 a 25 años manifestó una mayor prevalencia de $73.6 \%$ en el consumo de 
tabaco, y para ese mismo grupo etario existe una mayor prevalencia de vida en el consumo de alcohol $(87.2 \%)$.

Además, entre los estudiantes de los diferentes tipos de universidades que admitieron alguna vez haber consumido alcohol y tabaco, los de universidades particulares manifestaron un consumo mayor ante dichos productos $(90.5 \%$ y $73.1 \%$ respectivamente).

Se encontró que un gran porcentaje manifiesta una alta tendencia en las categorías de un nivel promedio de autoconcepto, $53.8 \%$, seguido de un nivel alto de $21.8 \%$, siendo estos datos importantes para nuestra investigación. A su vez, en la Tabla 1 se muestra el análisis sobre los niveles de autoconcepto que manifiestan los estudiantes universitarios dentro de cada uno de los componentes que forman este constructo, encontrándose que la gran mayoría de ellos, se ubica en una categoría media (promedio) en cada uno de los componentes: (académico $44.3 \%$, social $45.8 \%$, emocional $47.7 \%$, familiar $45.7 \%$ y físico $45.2 \%$ ).

En la Tabla 2 se muestran los coeficientes de correlación de Pearson para el autoconcepto y los factores protectores psicosociales obtenidos para la muestra total. Se observa en dicha Tabla que las correlaciones entre el autoconcepto y los factores psicosociales son moderadas pero altamente significativas y positivas, observándose que un alto autoconcepto se encuentra asociado con la existencia de un alto conocimiento y cumplimiento de normas familiares y escolares $(r=0.47 ; p<.01)$; cuanto mayor es el apoyo social mayor es el autoconcepto $(r=0.48$; $\mathrm{p}<.01$ ), asimismo cuanto mayor es el autoconcepto mayor es la valoración del cuerpo y de la vida $(\mathrm{r}=$ $0.43 ; \mathrm{p}<.01)$. Además, se puede apreciar que cuanto más alto es el apoyo familiar mayor es el autoconcepto $(r=0.44 ; p<.01)$. Finalmente, se observa que una alta satisfacción familiar (relaciones familiares de alta estabilidad y cohesión) se encuentra relacionada con la presencia de un autoconcepto alto $(r=0.25 ; p<.01)$.

Se observa en la Tabla 3, que las correlaciones entre los componentes del autoconcepto y los factores protectores son entre moderadas y débiles pero altamente significativas, observándose que el autoconcepto académico presenta mayor grado de relación con la valoración del cuerpo y de la vida (r
$=0.50 ; \mathrm{p}<0.01)$, es decir, la valoración del cuerpo y la vida está asociado con repercusiones positivas en el rendimiento académico. Asimismo, el apoyo social brindado a los estudiantes presenta una relación positiva con el componente social del autoconcepto $(\mathrm{r}=0.35 ; \mathrm{p}<.01)$, también el apoyo social muestra una mayor relación con el componente emocional $(\mathrm{r}=0.19 ; \mathrm{p}<.01)$ y el componente físico $(\mathrm{r}=0.38$; $\mathrm{p}<0.01)$. Además, se puede apreciar que un buen sistema de apoyo familiar mejorará y ayudará para que el autoconcepto familiar de los estudiantes sea elevado $(r=0.68 ; p<.01)$. Finalmente, se observa que cuanto mayor es la satisfacción familiar mayor es el autoconcepto familiar $(r=0.29 ; \mathrm{p}<.01)$.

En la Tabla 4, se presentan las correlaciones entre la frecuencia de consumo de alcohol y los componentes del autoconcepto. En este sentido observamos que, a excepción del componente emocional, todos los demás (académico, social, familiar y físico) presentan correlaciones altamente significativas y negativas con el consumo de alcohol $(\mathrm{r}=-0.28, \mathrm{p}<.01 ; \mathrm{r}=$ $-0.10, \mathrm{p}<.01 ; \mathrm{r}=-0.28, \mathrm{p}<.01 ; \mathrm{r}=-0.25, \mathrm{p}<.01$, respectivamente).

La Tabla 5 muestra las correlaciones entre la frecuencia de consumo de tabaco y los componentes del autoconcepto, observándose que los componentes académico, social, familiar y físico presentan una relación débil pero altamente significativa y negativa con el consumo de tabaco. Esto quiere decir que cuanto mayor desarrollo presentan los estudiantes en estos componentes, menor es la probabilidad de consumo.

De otra parte, también se observa que entre el componente emocional y el consumo de tabaco no existe relación significativa $(\mathrm{r}=-0.04, \mathrm{p}>.05)$.

La ecuación del modelo de Regresión Lineal Múltiple es significativo $(\mathrm{F}=14.438, \mathrm{p}<.01)$ para el consumo de alcohol (Tabla 6). Los factores del modelo explican en un $12 \%$ el consumo de alcohol en los estudiantes universitarios. En los pesos Beta estandarizados, valor para comparar el efecto de cada factor en el consumo de alcohol, se observa que la variable autoconcepto es el factor con mayor capacidad explicativa en los universitarios $(\beta=-0.203, p<.01)$. Además, se encontró que la valoración del cuerpo y de vida es la segunda variable con mayor peso explicativo en el consumo de alcohol $(\beta=-0.178, p<.01)$. Tanto 
el primer factor como los demás factores explicativos significativos presentan una relación negativa con el consumo de alcohol, lo cual quiere decir que cuanto mayor es el autoconcepto y mayor la valoración que se tiene hacia el cuidado del cuerpo y la salud menores son las probabilidades del consumo.

Podemos observar (Tabla 7), que la ecuación del modelo de Regresión Lineal Múltiple es significativo $(\mathrm{F}=8.494, \mathrm{p}<.01)$ para el consumo de tabaco. Los factores incluidos en el modelo explican en un $8 \%$ el consumo de tabaco en los universitarios. En los pesos Beta estandarizados, se observa que la variable valoración del cuerpo y de vida es el factor con mayor capacidad explicativa en los universitarios $(\beta=-0.165$, $\mathrm{p}<.01)$. Se observa además que el autoconcepto es la segunda variable con mayor peso explicativo en el consumo de tabaco $(\beta=-0.120, \mathrm{p}<.01)$.

Las variables autoconcepto y la valoración del cuerpo y de la vida se encuentran asociadas de manera significativa con el consumo de tabaco. Tanto el autoconcepto como la valoración que se tiene del cuerpo y de la vida se constituyen en factores de protección para el consumo de tabaco $(\mathrm{OR}=0.973$, $\mathrm{p}$ $<.05 ; \mathrm{OR}=0.913, \mathrm{p}<.01$ respectivamente $)$.

\section{DISCUSIÓN}

Un hallazgo importante se relaciona con la edad de inicio y prevalencia del consumo de alcohol y tabaco en los estudiantes de pregrado. Encontramos que la edad de inicio en el consumo de alcohol (4.8\%) y tabaco $(6.9 \%)$ se presenta a partir de los 8 y 9 años. En otro estudio llevado a cabo con una muestra de 4495 universitarios en Lima se encontró que los estudiantes habían iniciado el consumo de tabaco (1.9\%) y alcohol (6.8\%) antes de los 10 años (Mesa de Trabajo de Universidades [MTU], 2005); de modo similar Vallejos (2004) reporta en una muestra de escolares que la edad de inicio del consumo de alcohol y tabaco es antes de los 10 años. De otro lado, el Instituto especializado de salud mental "Honorio Delgado Hideyo Noguchi" en el 2002, indicaba que la edad de inicio del consumo de estas drogas se presentaba entre los 12 y 13 años (Saavedra et al., 2006).

Respecto a la prevalencia de vida en el consumo de alcohol y tabaco nuestros datos indican, que la sustancia más consumida por los estudiantes universitarios es el alcohol $(85,1 \%)$ y en segundo lugar es el tabaco $(69,1 \%)$. Este hallazgo se corrobora con el estudio realizado por Contradrogas-INEI (1998) y MTU (2005) quienes señalan índices altos en el consumo de estas sustancias, en comparación con otras drogas ilegales. En cuanto al consumo de alcohol, CEDRO (1999) señala que es la droga psicoactiva de mayor consumo, y la causante de trastornos biológicos, sociales, familiares, económicos y psicológicos. Es importante, resaltar que este estudio junto con diversas investigaciones de corte epidemiológico, presentan datos similares sobre las drogas en el Perú (Ferrando, 1992; Cedro, 1993 y 1997; ContradrogasINEI, 1998).

Otro hallazgo importante que evidencia nuestro estudio es que los estudiantes de universidades particulares presentaron mayor consumo de alcohol $(90,5 \%)$ y tabaco $(73.1 \%)$ en contraste a los estudiantes de las universidades públicas.

La Organización Mundial de la Salud (citado por Vallejos, 2004), señala que los problemas más importantes de salud en la etapa de la adolescencia son los que surgen de situaciones generadoras de riesgo.

Algunas investigaciones señalan que mientras existan factores de riesgo que se encuentren en el sistema social (escuela, familia, compañeros y comunidad), mayor será la tendencia hacia los problemas relacionados a la droga (Vallejos, 2004; Valdez, Labra y Lara, 1995; Volkow, 2004).

En nuestra investigación, los datos muestran evidencias que de las variables propuestas, el autoconcepto y la valoración del cuerpo y de la vida son factores protectores relevantes para el consumo de alcohol $(\mathrm{OR}=0.959, \mathrm{p}<0.01 ; \mathrm{OR}=0.905, \mathrm{p}<0.01$ respectivamente), es decir, que los estudiantes que presenten un buen autoconcepto de sí mismos y tengan una valoración positiva de sí mismos y de la vida, tendrán menores probabilidades de consumir alcohol. Así mismo, el autoconcepto y la valoración del cuerpo y de la vida se encuentran asociadas de manera significativa y se constituyen como factores de protección para el consumo de tabaco $(\mathrm{OR}=$ $0.973, \mathrm{p}<0.01 ;$ OR $=0.913, \mathrm{p}<0.01$ respectivamente $)$. Además, existen otros estudios realizados donde variables parecidas a la de nuestro estudio como el "apego familiar, el valorar la salud", y "el cumplimiento 
de leyes-normas de la comunidad" parecen proteger a los sujetos de determinadas edades en el consumo de drogas como el alcohol y el tabaco (Martínez y Robles, 2001).

\section{CONCLUSIONES}

1. Los estudiantes universitarios se iniciaron en el consumo de tabaco y alcohol entre los 8 y 9 años; presentándose mayor incremento en el inicio de consumo de ambas sustancias entre los 13 y 17 años (71.2\%). Y de los que alguna vez en su vida probaron, aproximadamente la mitad eran consumidores activos del alcohol $(46.2 \%)$ y tabaco $(30,8 \%)$ en el último mes de aplicada la encuesta.

2. Tanto en la prevalencia de vida, como en la del último mes de aplicada la encuesta, el mayor consumo de tabaco y alcohol recae en los estudiantes varones, con edades entre $21 \mathrm{y}$ 25 años, de padres separados y pertenecientes a universidades privadas.

3. El autoconcepto y los factores psicosociales tales como la valoración del cuerpo y la salud y la satisfacción familiar se encuentran asociados de manera negativa tanto con el consumo de alcohol como el tabaco en los estudiantes universitarios de la ciudad de Lima.

4. Mientras que, el factor de mayor peso explicativo en el consumo de alcohol es el autoconcepto, el factor de mayor capacidad explicativa en el consumo de tabaco es la valoración del cuerpo y de la vida.

5. De acuerdo a los valores OR (Odds Ratio) sólo el autoconcepto y la valoración del cuerpo y de la vida se constituyen en factores de protección para el consumo de alcohol y tabaco en los estudiantes universitarios.

\section{REFERENCIAS}

Bringas, J. (2002). Alcoholismo y familia. Monografía para optar el título de Licenciado en Psicología. Universidad Nacional Federico Villarreal. Lima, Perú.
Centro de Información y Educación para la Prevención del Abuso de Drogas, CEDRO (1993). Opiniones sobre drogas en el Perú. Población urbana. Monografía de Investigación $N^{\circ}$ 8. Lima: Autor.

Centro de Información y Educación para la Prevención del Abuso de Drogas, CEDRO (1997). Epidemiología del abuso de drogas en la población urbana de Lima. Monografía de Investigación $\mathrm{N}^{\circ}$ 12. Lima: Autor.

Centro de Información y Educación para la Prevención del Abuso de Drogas, CEDRO (1999). Factores de riesgo y de Protección en el abuso de drogas ilegales en adolescentes jóvenes de Lima Metropolitana. Perú: Autor.

CONTRADROGAS-INEI (1998). Resumen ejecutivo: Encuesta nacional de prevención y uso de drogas. Lima: Contradrogas.

Ferrando, D. (1992). Conocimiento y uso de drogas en los colegios secundarios. Encuesta nacional 1992. Lima: Ministerio de Educación.

García, F. y Musitu, G. (1999). Autoconcepto Forma 5 (AF5). Madrid: TEA.

Hernández, R.; Fernández, C. y Baptista, P. (1998). Metodología de la Investigación. México: Mc Graw Hill.

Kerlinger, F. y Lee, H. (2002). Investigación del comportamiento, métodos de investigación en ciencias sociales. México: Mc Graw Hill.

Kuri, P. (1994). Tabaquismo pasivo. En R. Tapia (Comp.), Las adicciones: dimensión, impacto $y$ perspectivas (pp. 113-122). México, D.F.: Manual Moderno.

Machargo, J. (1991). El profesor y el autoconcepto de sus alumnos. Madrid: Escuela Española.

Martínez, G. y Robles, L. (2001). Variables de protección ante el consumo de alcohol y tabaco en adolescentes. Psicothema, 13(2), 222 - 228.

Mesa de Trabajo de Universidades - MTU (2005). I encuesta sobre información, hábitos y actitudes hacia el consumo de sustancias psicoactivas en universitarios de Lima Metropolitana. Lima: DEVIDA.

Muñoz-Rivas, M. y Graña, J. (2001). Factores familiares de riesgo y de protección para el 
consumo de drogas en adolescentes. Psicothema, 13(1), 87-94.

Musitu, G., García, J. y Gutiérrez, M. (1991). AFA. Autoconcepto Forma A. Madrid, España: TEA.

Saavedra, J., Nizama, M. y Kendall, R. (2006). Estudio epidemiológico de salud mental en frontera 2005. Informe general. Anales de Salud Mental, 23(1), 33. Recuperado de http://es.scribd. com/doc/66947139/Anales-de-Salud-Mentalen-Fronteras-2005-INSM-HD-HN-Peru\#outer_ page_35

Tapia, C. (2001). Las adicciones, dimensión, impacto $y$ perspectivas $\left(2^{\mathrm{a}}\right.$ ed.). México (DF): Manual Moderno.

Tomás, J. y Oliver, A. (2004). Análisis psicométrico confirmatorio de una medida multidimensional del autoconcepto en español. Revista Interamericana de Psicología, 38(2), 285-293.

Valdez, M., Labra, J. y Lara, P. (1995). Factores familiares protectores para conductas de riesgo: Vulnerabilidad y resiliencia adolescentes. Revista psiquiatría clínica, 4(2), 49-56.

Vallejos, J. (2004). Consumo de drogas y factores de riesgo y protección en escolares de educación secundaria. Lima: DEVIDA.

Volkow, N. (2004). Cómo prevenir el uso de drogas en los niños y los adolescentes. National Institute on Drug Abuse (NIDA), 4, 142-175. Recuperado de http://www.nida.nih.gov/ Prevention/Spanish/ capitulo1.html 
Tabla 1

Niveles de autoconcepto en universitarios, según sus componentes

\begin{tabular}{llll}
\hline Componentes & $\begin{array}{c}\text { Niveles de } \\
\text { autoconcepto }\end{array}$ & Frecuencia & Porcentaje \\
\hline \multirow{3}{*}{ Académico } & Bajo & 159 & $22.6 \%$ \\
& Medio & 312 & $44.3 \%$ \\
& Alto & 234 & $33.2 \%$ \\
Social & Bajo & 164 & $23.3 \%$ \\
& Medio & 323 & $45.8 \%$ \\
Emocional & Alto & 218 & $30.9 \%$ \\
& & & \\
& Bajo & 170 & $24.1 \%$ \\
Familiar & Medio & 336 & $47.7 \%$ \\
& Alto & 199 & $28.2 \%$ \\
& Bajo & 170 & $24.1 \%$ \\
Físico & Medio & 322 & $45.7 \%$ \\
& Alto & 213 & $30.2 \%$ \\
& Bajo & 159 & $22.6 \%$ \\
& Medio & 319 & $45.2 \%$ \\
& Alto & 227 & $32.2 \%$ \\
\hline
\end{tabular}

Tabla 2

Coeficientes de correlación entre autoconcepto y factores protectores

\begin{tabular}{lll}
\hline Factores Psicosociales & \multicolumn{2}{c}{ Autoconcepto } \\
& $\mathrm{r}$ & $\mathrm{P}$ \\
\hline Normas familiares y escolares & $0.47^{* *}$ & 0.000 \\
Apoyo social & $0.48^{* *}$ & 0.000 \\
Valoración del cuerpo y de la vida & $0.43^{* *}$ & 0.000 \\
Apoyo familiar & $0.44^{* *}$ & 0.000 \\
Satisfacción familiar & $0.25^{* *}$ & 0.000 \\
\hline
\end{tabular}

** La correlación es significante al nivel 0.01 
Tabla 3

Relación entre los componentes del autoconcepto y los factores protectores

\begin{tabular}{lccccccccccc}
\hline \multirow{2}{*}{ Factores Psicosociales } & \multicolumn{2}{c}{ Académico } & \multicolumn{2}{c}{ Social } & \multicolumn{2}{c}{ Emocional } & \multicolumn{2}{c}{ Familiar } & \multicolumn{2}{c}{ Físico } \\
& $\mathrm{r}$ & $\mathrm{p}$ & $\mathrm{r}$ & $\mathrm{p}$ & $\mathrm{r}$ & $\mathrm{p}$ & $\mathrm{r}$ & $\mathrm{p}$ & $\mathrm{r}$ & $\mathrm{p}$ \\
\hline Normas familiares y escolares & $.44 * *$ & .000 & $.32 * *$ & .000 & $.12 * *$ & .001 & $.39 * *$ & .000 & $.37 * *$ & .000 \\
Apoyo Social & $.43 * *$ & .000 & $.35 * *$ & .000 & $.19 * *$ & .000 & $.30 * *$ & .000 & $.38^{* *}$ & .000 \\
Valoración del cuerpo y la vida & $.50 * *$ & .000 & $.26 * *$ & .000 & $.09 * *$ & .006 & .33 & .000 & $.34 * *$ & .000 \\
Apoyo Familiar & $.26 * *$ & .000 & $.13 * *$ & .000 & $.12 * *$ & .001 & $.68^{* *}$ & .000 & $.26 * *$ & .000 \\
Satisfacción Familiar & $.21 * *$ & .000 & $.22 * *$ & .000 & -.03 & .216 & $.29 * *$ & .000 & $.17 * *$ & .000 \\
\hline
\end{tabular}

** La correlación es significante al nivel 0.01 (unilateral)

Tabla 4

Coeficientes de correlación para componentes del autoconcepto y el consumo de alcohol

\begin{tabular}{llc}
\hline $\begin{array}{l}\text { Componentes del } \\
\text { autoconcepto }\end{array}$ & \multicolumn{2}{c}{ Consumo de alcohol } \\
\hline Académico & $-0.28 * *$ & $\mathbf{p}$ \\
Social & $-0.10 * *$ & 0.000 \\
Emocional & $-0.05 \mathrm{~ns}$ & 0.005 \\
Familiar & $-0.28 * *$ & 0.101 \\
Físico & $-0.25 * *$ & 0.000 \\
\hline
\end{tabular}

** La correlación es significante al nivel 0.01

ns La correlación no es significante $(\mathrm{p}>.05)$

Tabla 5

Coeficientes de correlación para componentes del autoconcepto y el consumo de tabaco

\begin{tabular}{lcc}
\hline $\begin{array}{l}\text { Componentes del } \\
\text { autoconcepto }\end{array}$ & \multicolumn{2}{c}{ Consumo de tabaco } \\
\hline Académico & $-0.22 * *$ & $\mathbf{p}$ \\
Social & $-0.10 * *$ & 0.000 \\
Emocional & $-0.04 \mathrm{~ns}$ & 0.009 \\
Familiar & $-0.20 * *$ & 0.164 \\
Físico & $-0.15 * *$ & 0.000 \\
\multicolumn{2}{c}{$* *$ La correlación es significante al nivel .01 } & 0.000 \\
\multicolumn{2}{c}{ ns La correlación no es significante $(\mathrm{p}>.05)$} \\
\end{tabular}


Tabla 6

Análisis de Regresión Múltiple para la variable dependiente consumo de alcohol

\begin{tabular}{|c|c|c|c|c|c|c|c|}
\hline \multirow[t]{2}{*}{ Variables } & \multicolumn{2}{|c|}{$\begin{array}{l}\text { Coeficientes no } \\
\text { estandarizados }\end{array}$} & \multirow{2}{*}{$\begin{array}{c}\text { Coeficientes } \\
\text { estandarizados } \\
\text { Beta }\end{array}$} & \multirow[t]{2}{*}{$\mathbf{t}$} & \multirow[t]{2}{*}{ Sig. } & \multicolumn{2}{|c|}{$\begin{array}{l}\text { Estadísticos } \\
\text { de colinealidad }\end{array}$} \\
\hline & B & Error típ. & & & & Tolerancia & FIV \\
\hline (Constante) & 2.221 & .205 & & 10.839 & .000 & & \\
\hline Autoconcepto & $-9.280 \mathrm{E}-03$ & .002 & -.203 & -4.153 & .000 & .601 & 1.664 \\
\hline Apoyo familiar & $-3.887 \mathrm{E}-02$ & .025 & -.068 & -1.576 & .115 & .774 & 1.293 \\
\hline $\begin{array}{l}\text { Normas familiares } \\
\text { y escolares }\end{array}$ & $-3.343 \mathrm{E}-03$ & .010 & -.016 & -.337 & .736 & .642 & 1.558 \\
\hline Satisfacción familiar & $-2.296 \mathrm{E}-02$ & .010 & -.103 & -2.406 & .016 & .784 & 1.275 \\
\hline Apoyo social & $-1.265 \mathrm{E}-03$ & .008 & -.008 & -.150 & .881 & .571 & 1.752 \\
\hline $\begin{array}{l}\text { Valoración del cuerpo } \\
\text { y la vida }\end{array}$ & $-2.285 \mathrm{E}-02$ & .006 & -.178 & -3.664 & .000 & .607 & 1.649 \\
\hline
\end{tabular}

Tabla 7

Análisis de Regresión Múltiple para la variable dependiente consumo de tabaco

\begin{tabular}{|c|c|c|c|c|c|c|c|}
\hline \multirow[t]{2}{*}{ Factores } & \multicolumn{2}{|c|}{$\begin{array}{l}\text { Coeficientes no } \\
\text { estandarizados }\end{array}$} & \multirow{2}{*}{$\begin{array}{l}\text { Coeficientes } \\
\text { estandarizados } \\
\text { Beta }\end{array}$} & \multirow[t]{2}{*}{$\mathbf{t}$} & \multirow[t]{2}{*}{ Sig. } & \multicolumn{2}{|c|}{$\begin{array}{l}\text { Estadísticos de } \\
\text { colinealidad }\end{array}$} \\
\hline & B & Error típ. & & & & Tolerancia & FIV \\
\hline (Constante) & 1.584 & .204 & & 7.777 & .000 & & \\
\hline Autoconcepto & $-5.29 \mathrm{E}-03$ & .002 & -.120 & -2.373 & .018 & .604 & 1.655 \\
\hline Apoyo familiar & $-4.26 \mathrm{E}-02$ & .024 & -.080 & -1.794 & .073 & .783 & 1.277 \\
\hline $\begin{array}{l}\text { Normas familiares } \\
\text { y escolares }\end{array}$ & 4.280E-03 & .010 & .022 & .442 & .659 & .645 & 1.551 \\
\hline Satisfacción familiar & $-1.548 \mathrm{E}-02$ & .009 & .074 & 1.681 & .093 & .791 & 1.264 \\
\hline Apoyo social & $-5.72 \mathrm{E}-03$ & .008 & -.036 & -.701 & .484 & .570 & 1.754 \\
\hline $\begin{array}{l}\text { Valoración del cuerpo } \\
\text { y la vida }\end{array}$ & $-1.98 \mathrm{E}-02$ & .006 & -.165 & -3.239 & .001 & .592 & 1.690 \\
\hline
\end{tabular}

Fecha de recepción: 20 de agosto, 2011

Fecha de aceptación: 24 de octubre, 2011 